\title{
Karl Frei \\ Genes and pathways driving glioblastomas in humans and murine disease models
}

Published online: 29 May 2003

(C) Springer-Verlag 2003

This scholarly review describes characteristic genetic alterations that underlie the initiation and progression of glioblastomas in humans and rodent models. These alterations include the inactivation of tumor suppressor genes such as TP53, CDKN2A/p16, RB, and PTEN and activation of oncogenes including the epidermal growth factor receptor (EGFR), CDK4, CDK6, Ras, and Akt.

In the first part of the review, the embryonic development of the brain is addressed, followed by two hypotheses on the cellular origin of gliomagenesis. With the identification and characterization of a CNS stem cell that persists into adulthood, the first hypothesis that glioma represents transformation of a neural stem cell within the subventricular zone has gained attractiveness for brain tumor researchers. The second hypothesis is more conventional, assuming that gliomas arise by transformation of an intraparenchymal glial cell that forms a mass which expands centrifugally, invading the surrounding tissue. The therapeutic implications of neural stem cells are also discussed, but at the moment it is not yet clear whether this approach will prove of clinical value.

In the next part of the review, the major pathways of gliomagenesis such as the growth factors (EGFR, PDGFR), components of the cell-cycle machinery ( $\mathrm{Rb}$, cdk4, and the cdk inhibitor p16), and regulators of apoptosis (p53, ARF, and PTEN) are discussed in detail and illustrated with the aid of a series of graphics. In addition, to determine the importance of altered pathways, the current mouse models of glioma formation are described.

Next, the new and strong experimental evidence supporting the concept of a mutator phenotype in cancer is reviewed, including potential genes that might drive a glioma mutator phenotype.

Finally, glioma invasiveness is discussed as a key factor of glial malignancy from the level of growth factors and Rac-dependent migration. Moreover, data on focal adhesion kinase (FAK) activity and its role in the extent and dynamics of invasiveness are given. A scheme illustrates the link between migration and survival or anoikis and the use of several alternating pathways in gliomas.

Overall, this review is intellectually stimulating and demonstrates that the mysteries of gliomagenesis are being unraveled. However, several questions remain whose answers will be extremely helpful in understanding the biology of human gliomas and designing successful therapies for them in the future.

\author{
K. Frei ( $)$ \\ Department of Neurosurgery, \\ University Hospital of Zürich, \\ Frauenklinikstrasse 10, 8091 Zürich, Switzerland \\ e-mail: karl.frei@usz.ch \\ Tel.: +41-1-2552479 \\ Fax: +41-1-2554514
}

